\title{
The Jeans modeling of the Milky Way galaxy: implications of the kinematics of the stellar halo
}

\begin{abstract}
S. Samurović and A. Lalović
Astronomical Observatory, Volgina 7, 11160 Belgrade, Serbia

e-mail: [srdjan;ana]@aob.bg.ac.rs

Received 17 March 2011 / Accepted 28 April 2011

ABSTRACT

Aims. We investigate the predictions of Newtonian dynamics and the MOND theory related to the Milky Way galaxy using the Jeans equation.

Methods. We used the measurements of the radial velocities of the blue horizontal branch (BHB) halo stars to test the predictions of Newtonian gravity and to also extend our study to different MOND models, taking orbital anisotropies that we calculate into account. Results. The halo stars of the Galaxy were used as a tracer of the Galaxy's gravitational potential. The Jeans equation was calculated for both the Newtonian and the MOND approaches. We assumed spherical symmetry and calculated the Jeans equation by taking orbital anisotropies into account. Circular velocities for both approaches were also analyzed.

Conclusions. We solved the Jeans equation in spherical approximation and confirm that the Newtonian model without dark matter cannot fit the observed velocity dispersion profile and that the truncated flat model with dark matter can provide a good fit to the observed velocity dispersion. For the MOND models, from the Jeans modeling and the models of the circular velocity curves, we found that two models can provide a fit to the data without significant anisotropies whereas two other tested models need various anisotropies to obtain the same result.
\end{abstract}

Key words. gravitation - galaxies: spiral - Galaxy: kinematics and dynamics - Galaxy: halo

\section{Introduction}

The mass profile and the problem of dark matter in the Milky Way galaxy are frequently discussed in the literature (some recent papers include: Sakamoto et al. 2003; Battaglia et al. 2005; Gnedin et al. 2010; see also an innovative review of the issue of dark matter in galaxies by Salucci et al. 2011). Since the tracers of rotation of the disk, both stars and gas, extend out to $20 \mathrm{kpc}$ (Sofue et al. 2009), various alternatives have been sought. These alternatives are based on solving of the Jeans equation (e.g. Binney \& Tremaine 2008), in which one models the profile of radial velocity dispersion of the tracers of the mass of the Galaxy. For example, Battaglia et al. (2005) compiled a sample of 240 halo objects with accurate distances and radial velocities, and their sample provides insight into kinematics of the heterogeneous sample that consists of globular clusters (GCs), satellite galaxies, field blue horizontal branch (BHB) stars, and red giants. They find that the dispersion falls from $\sim 120 \mathrm{~km} \mathrm{~s}^{-1}$ in the central parts (between 10 and $20 \mathrm{kpc}$ ) to $\sim 50 \mathrm{~km} \mathrm{~s}^{-1}$ (beyond $\sim 100 \mathrm{kpc}$ ). More recently, several interesting attempts have also been made that addressed the problem using various tracers. Xue et al. (2008, hereafter Xue08) derived new constraints on the mass of the dark matter halo using a sample of 2401 carefully selected BHB stars taken from SDSS DR6. They found within a radius of $60 \mathrm{kpc}$ the total mass of the Milky Way equal to $(4.0 \pm 0.7) \times 10^{11} M_{\odot}$. The profile of the velocity dispersion that they found is rather flat (varies between 90 and $115 \mathrm{~km} \mathrm{~s}^{-1}$ throughout the whole studied region). More recently, Brown et al. (2010, hereafter Bro10) have used their sample of 910 distant halo stars from the Hypervelocity star survey to also derive the velocity dispersion profile of the Galaxy. Their sample includes $74 \%$ of evolved BHB stars and $26 \%$ blue stagglers, and it contains twice as many objects beyond $\sim 50 \mathrm{kpc}$ as previous surveys. They find that the Milky Way shows a mean decline in radial velocity dispersion of $-0.38 \pm 0.12 \mathrm{~km} \mathrm{~s}^{-1} \mathrm{kpc}^{-1}$ over $15<R<75 \mathrm{kpc}$. The results of Xue 08 were used in the paper by McGaugh (2008), who modeled the outer rotation curve of the Milky Way using MOND. He finds that MOND (Milgrom 1983) naturally provided good match to the rotation curve and that dark matter is not needed within this approach. On the other hand, the results of Bro10 were used in the paper by Gnedin et al. (2010) to find that the total mass of the Milky Way within $80 \mathrm{kpc}$ is $6.9_{-1.2}^{+3.0} \times 10^{11} M_{\odot}$ and that the observed approximately constant velocity dispersion profile requires a massive and extended dark halo.

One important aspect of modeling the total mass of some galaxy (and of course, the Galaxy, as well) is the problem of anisotropies in the motion of the chosen tracers because of the well-known mass-orbital anisotropy degeneracy (Tonry 1983). The aforementioned authors in their papers addressed this problem in various ways. Battaglia et al. (2005) used toy models based on $N$-body $\Lambda$ CDM models and proper motion measurements of the nearby dwarf spheroidals, Xue 08 used cosmologically motivated galaxy simulations and then produced "mock observations" with these simulations to match test results to the observations and Gnedin et al. (2010) took the predictions of cosmological simulations of galaxy formation as a guide for their modeling purposes. In the present work we have adopted a different strategy: by using standard statistical approach (see below), from the observed radial velocities of BHB stars in our 
sample to calculate radial velocity dispersion but also skewness and kurtosis, which provide information about anisotropies of the motion of the tracers.

It is worth noting that the methodology of establishing the existence of dark matter using the Jeans equation (for both the Newtonian and the MOND approach) for various types of tracers, such as stars, planetary nebulae (PNe), and GCs is well tested in the case of early-type (elliptical) galaxies (see for example Romanowsky et al. 2003; Peng et al. 2004; Richtler et al. 2004; Samurović \& Danziger 2005, 2006; Samurović \& Ćirković 2008; Samurović 2010). The decrease in the velocity dispersion was taken as an indication of the lack of dark matter in the outer regions of various early-type galaxies. Recently, Napolitano et al. (2011) have obtained the data for $\sim 450 \mathrm{PNe}$ for NGC 4374 and the approximate flatness of the velocity dispersion profile (together with the negligible kurtosis parameter) indicated for the first time a case where PNe dynamics is consistent with a standard dark matter halo.

The plan of the paper is as follows. In Sect. 2 we present the observational data related to the sample we constructed and discuss the kinematics of the sample, in Sect. 3 we solve the Jeans equation in both the Newtonian and the MOND approaches and present the circular curves for both approaches, and finally in Sect. 4 we draw our conclusions.

\section{Observational data}

\subsection{Halo stars of the Milky Way}

We used the same sample of stars as Xue08 along with those in Bro10 not already included from Xue08. The aim was to secure more stars in the outer region of the Milky Way (outside $60 \mathrm{kpc}$ ). In total we have 2557 stars that are found out to $85 \mathrm{kpc}: 2416$ from Xue08 and 141 from Bro10. All these stars were selected to satisfy the following criteria: i) they must have $|z|>4 \mathrm{kpc}$ in order to exclude thick-disk stars; ii) radial velocity error is less than $30 \mathrm{~km} \mathrm{~s}^{-1}$; and iii) they are all BHB stars. (The likelihood of being BHB star is $f=1$ in Bro10, Xue 08 contains only BHB stars.)

The kinematics of the Milky Way is given in Fig. 1 and in Table 1. In the top panel of Fig. 1 we present radial velocities of the stars in the Galactic standard of rest in the sample, and below we present the radial velocity dispersion $\sigma_{r}$, the skewness $s_{3}$, and kurtosis $s_{4}$, which we calculated using standard definitions (see below). The profile of the velocity dispersion remains approximately constant (within the error bars) throughout the whole observed region.

The third moment $s_{3}$ describes the asymmetric departures from the Gaussian:

$s_{3}=\frac{1}{N} \sum_{i=1}^{N}\left[\frac{x_{i}-\bar{x}}{\sigma}\right]^{3}$.

Detecting a positive (negative) value of the $s_{3}$ parameter would mean that the distribution is skewed towards higher (lower) velocities with respect to the systemic velocity.

The fourth moment $s_{4}$ describes symmetric departures from the Gaussian:

$s_{4}=\frac{1}{N} \sum_{i=1}^{N}\left[\frac{x_{i}-\bar{x}}{\sigma}\right]^{4}-3$.

If one detects $s_{4}>0$, then the distribution is more peaked than the Gaussian at low velocities with more extended high-velocity

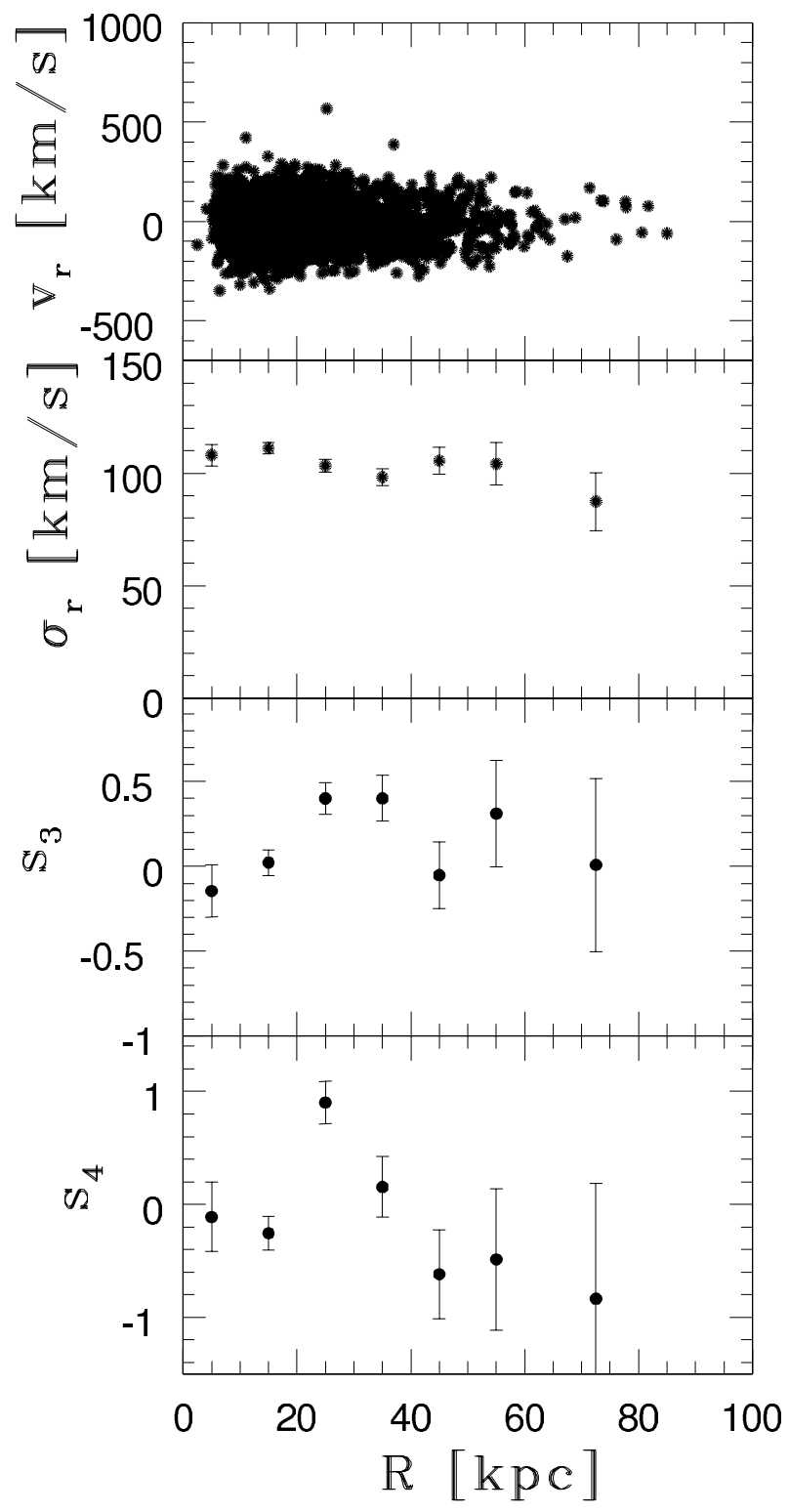

Fig. 1. Kinematics of the Milky Way BHB stars. From top to bottom: radial velocity of the stars in $\mathrm{km} \mathrm{s}^{-1}$, radial velocity dispersion calculated in a given bin with $s_{3}$ and $s_{4}$ parameters, which describe symmetric and asymmetric departures from the Gaussian, respectively.

tails, and for $s_{4}<0$ the distribution is more flat-topped than the Gaussian.

The errors for these statistical quantities were calculated using standard expressions found in Kendall \& Stuart (1977). It is important to stress that in our analysis based on the Jeans equation we use reduced kurtosis, which is important in breaking up the mass-orbital anisotropy degeneracy. From Fig. 1 one can see that $s_{3}$ is either consistent with zero or is moderately positive, whereas the $s_{4}$ parameter is consistent with zero and moderately negative, apart from one point at $25 \mathrm{kpc}$ (this hints at tangential anisotropies).

\subsection{Rotation of the halo stars}

The problem of the rotation of halo populations in the Milky Way and its importance has recently been reviewed in Deason et al. (2011). Since in the Jeans equation that we solve in Sect. 3 
Table 1. Kinematics data for BHB stars of the Milky Way in the galactocentric frame.

\begin{tabular}{lccccccc}
\hline \hline $\begin{array}{l}\langle r\rangle \\
(\mathrm{kpc})\end{array}$ & $\begin{array}{c}\sigma_{r} \\
\left(\mathrm{~km} \mathrm{~s}^{-1}\right)\end{array}$ & $\begin{array}{c}\text { err_o } \sigma_{r} \\
\left(\mathrm{~km} \mathrm{~s}^{-1}\right)\end{array}$ & $s_{3}$ & err_s & $s_{4}$ & err_s $s_{4}$ & $N$ \\
$(1)$ & $(2)$ & $(3)$ & $(4)$ & $(5)$ & $(6)$ & $(7)$ & $(8)$ \\
\hline 5 & 108.08 & 4.79 & -0.14 & 0.15 & -0.11 & 0.31 & 255 \\
15 & 111.13 & 2.42 & 0.02 & 0.08 & -0.26 & 0.15 & 1052 \\
25 & 103.33 & 2.80 & 0.40 & 0.09 & 0.90 & 0.19 & 680 \\
35 & 98.25 & 3.82 & 0.40 & 0.13 & 0.15 & 0.27 & 331 \\
45 & 105.55 & 5.99 & -0.05 & 0.20 & -0.62 & 0.40 & 155 \\
55 & 104.14 & 9.43 & 0.31 & 0.31 & -0.49 & 0.63 & 61 \\
73 & 87.42 & 12.89 & 0.01 & 0.51 & -0.83 & 1.02 & 23 \\
\hline
\end{tabular}

Notes. Column (1): central point for a given bin. Column (2): radial velocity dispersion for the sample of BHB stars in a given bin. Column (3): formal errors for the radial velocity dispersion of the sample of BHB stars. Column (4): $s_{3}$ parameter for the sample of BHB stars in a given bin. Column (5): formal errors for the $s_{3}$ parameter for the sample of BHB stars. Column (6): $s_{4}$ parameter for the sample of BHB stars in a given bin. Column (7): formal errors for the $s_{4}$ parameter for the sample of BHB stars. Column (8): number of BHB stars in a given bin.

Table 2. Rotation amplitude for BHB stars of the Milky Way in the Galactocentric frame.

\begin{tabular}{lccrrrrr}
\hline \hline $\begin{array}{l}r_{\text {in }} \\
(\mathrm{kpc})\end{array}$ & $\begin{array}{c}r_{\text {out }} \\
(\mathrm{kpc})\end{array}$ & $\begin{array}{c}V_{\text {sys }} \\
\left(\mathrm{km} \mathrm{s}^{-1}\right) \\
(3)\end{array}$ & $\begin{array}{c}\mathrm{err} V_{\text {sys }} \\
(2)\end{array}$ & $\begin{array}{c}A_{\text {rot }} \\
(4)\end{array}$ & $\begin{array}{c}\operatorname{err} A_{\text {rot }} \\
\left(\mathrm{km} \mathrm{s}^{-1}\right) \\
(5)\end{array}$ & $\begin{array}{c}f^{\prime} \\
(6)\end{array}$ & $N$ \\
\hline 0.00 & 13.99 & -6.74 & 4.25 & 0.50 & 5.94 & 0.712 & 634 \\
14.00 & 19.99 & -0.36 & 7.40 & 1.47 & 10.4 & 0.513 & 673 \\
20.00 & 27.99 & -3.46 & 5.37 & -6.56 & 7.58 & 0.497 & 586 \\
28.00 & 85.00 & -15.1 & 3.46 & -8.39 & 4.88 & 0.468 & 664 \\
\hline
\end{tabular}

Notes. Column (1): inner point for a given bin (in kpc). Column (2): outer point for a given bin (in kpc). Column (3): systemic velocity of the stars in a given bin (in $\mathrm{km} \mathrm{s}^{-1}$ ). Column (4): error for the systemic velocity of the stars in a given bin (in km s${ }^{-1}$ ). Column (5): rotation amplitude of the stars in a given bin (in $\mathrm{km} \mathrm{s}^{-1}$ ). Column (6): error for the rotation amplitude of the stars in a given bin (in $\mathrm{km} \mathrm{s}^{-1}$ ). Column (7): $f^{\prime}$ fraction (see text for details). Column (8): number of BHB stars in a given bin.

there is also a rotational term (see Eq. (4) below), we calculated the rotation of BHB stars in the sample.

The procedure consists of fitting a sine-curve to the velocities as the function of the position angle $\Theta$ :

$v_{\mathrm{r}}=V_{\mathrm{sys}}+A_{\mathrm{rot}} \sin (\Theta)$,

where $V_{\text {sys }}$ is the systemic velocity and $A_{\text {rot }}$ rotation amplitude and the fitting is done in four bins with approximately the same number of stars per bin. The position angle $\Theta$ is measured in the plane of the Milky Way between the direction towards the Sun and the projection of the star's position onto the Galactic plane. It ranges between -180 and 180 degrees and is measured positive in the clockwise direction as seen from the north Galactic pole. The results are given in Table 2 and Fig. 2.

As in the paper by Schuberth et al. (2010), we calculated the rotation amplitude for 1000 Monte Carlo realizations by keeping the positions of our original dataset while permuting the velocities. Thus, we obtained the fraction $f^{\prime}$ (prime in the exponent avoids confusion with the parameter $f$ mentioned in Sect. 2.1) of Monte Carlo runs for which a rotation amplitude is larger than the observed one. Therefore, the lower value of $f^{\prime}$ means the higher significance of the rotation signal. In Table 2 it can be seen that the rotation amplitude is small and consistent with zero in the first three bins, whereas in the last, wide bin there is an indication of the non-zero rotational signal ( $f^{\prime}$ fraction is also less than in the first three bins), $A_{\text {rot }}=-8.39 \pm 4.88 \mathrm{~km} \mathrm{~s}^{-1}$.

\section{Dynamical models}

We solve the spherically symmetric Jeans equation (e.g. Binney \& Tremaine 2008),

$\frac{\mathrm{d} \sigma_{r}^{2}}{\mathrm{~d} r}+\sigma_{r}^{2} \frac{\left(2 \beta_{*}+\alpha\right)}{r}=a_{N ; M}+\frac{v_{\mathrm{rot}}^{2}}{r}$,

in both the Newtonian and the MOND approaches and $a_{\mathrm{N} ; \mathrm{M}}$ is an acceleration term, that is equal to $a_{\mathrm{N}}=-\frac{G M(r)}{r^{2}}$ for Newtonian ("N") models and for MOND ("M") models $a_{\mathrm{M}} \mu\left(a_{\mathrm{M}} / a_{0}\right)=a_{\mathrm{N}}$ (see Sect. 3.2 below for details regarding function $\mu$ ), and $\sigma_{r}$ is the radial stellar velocity dispersion, $\alpha=\mathrm{d} \ln \rho / \mathrm{d} \ln r$ is the slope of tracer density $\rho$ and is taken to be $\alpha=-4$ (see Gnedin et al. 2010, for details). We also make use of the following equation for the acceleration (e.g. Binney \& Tremaine 2008):

$\frac{\mathrm{d} \Phi}{\mathrm{d} r}=\frac{G M(r)}{r^{2}}=\frac{V_{\mathrm{c}}^{2}}{r}$,

where $V_{\mathrm{c}}$ is the circular velocity. As shown above the rotation speed $v_{\text {rot }}$ of BHB stars is either consistent with zero or is very small and will be neglected in all modeling below.

The nonspherical nature of the stellar velocity dispersion is described by the $\beta_{*}$ parameter:

$\beta_{*}=1-\frac{\overline{v_{\theta}^{2}}}{\sigma_{r}^{2}}$,

where $\bar{v}_{\theta}^{2}={\overline{v_{\theta}}}^{2}+\sigma_{\theta}^{2}$. For $0<\beta_{*}<1$, the orbits are predominantly radial (equivalent to $s_{4}>0$ ), and the radial velocity distribution 


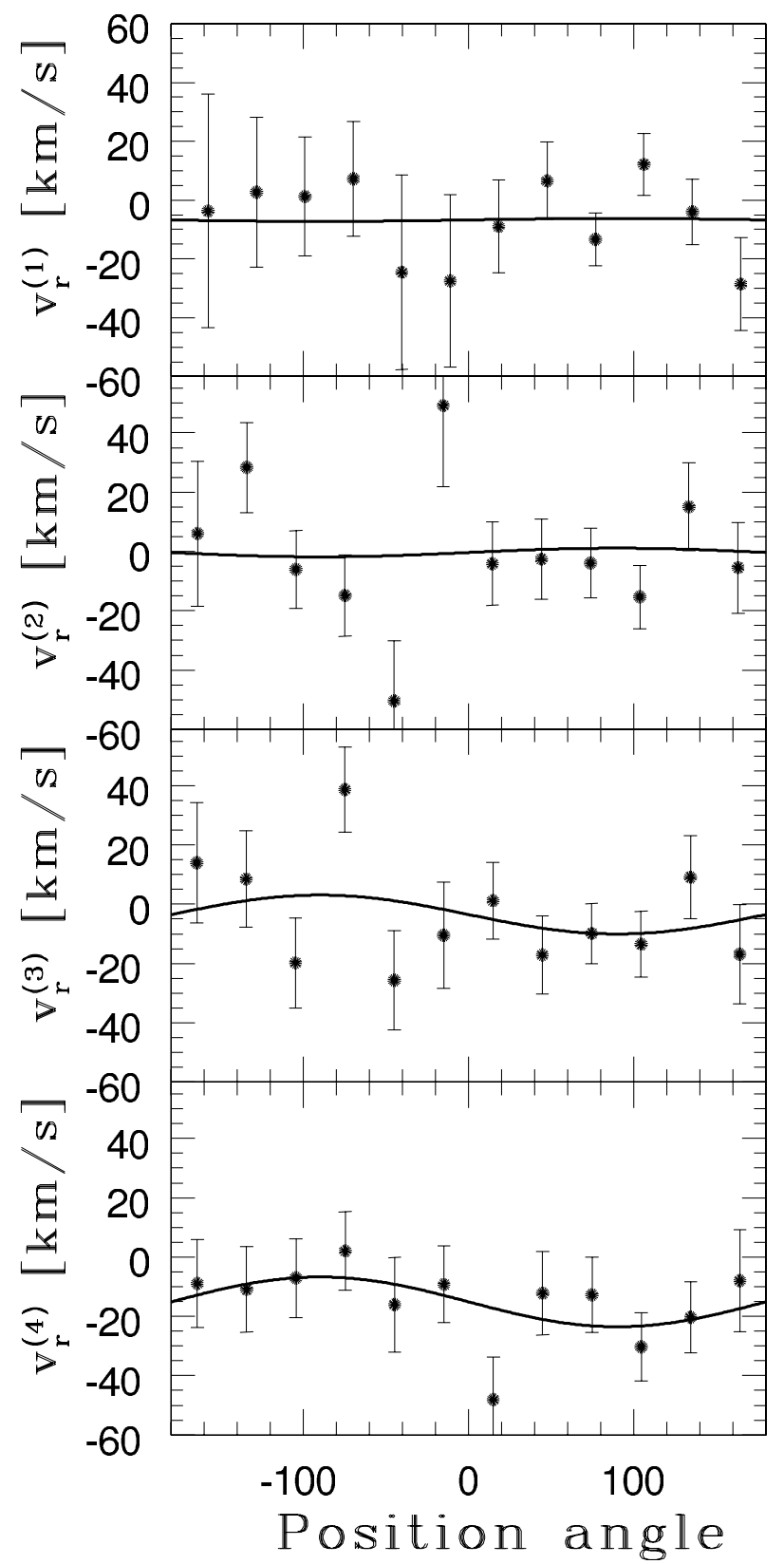

Fig. 2. Rotation of BHB stars as a function of the position angle $\Theta$ in 4 bins. From top to bottom, bins are sorted in the increasing order of galactocentric radii (the bin labeled "(1)" is the innermost and the bin labeled "(4)" is the outermost region). The thick solid line represents a fit obtained using Eq. (3).

is more strongly peaked than a Gaussian profile. On the other hand, the orbits are mostly tangential for $-\infty \leq \beta_{*}<0$ (equivalent to $s_{4}<0$ ), so that the profile is more flat-topped than a Gaussian (Gerhard 1993). In our models we used the $\gamma=4$ panel of Fig. 8 of Gerhard (1993) to convert from the observed kurtosis to the intrinsic anisotropy. We consider the Poisson errors of kurtosis and the systematic error of the conversion method. We realize that this conversion is strictly valid only for external galaxies and scale-free systems. It would require highly significant theoretical modeling to improve this. Nevertheless the resulting anisotropy profile (inset of Fig. 3) is not far from the standard isotropy assumption $\left(\beta_{*}=0\right)$ and has a large error bar

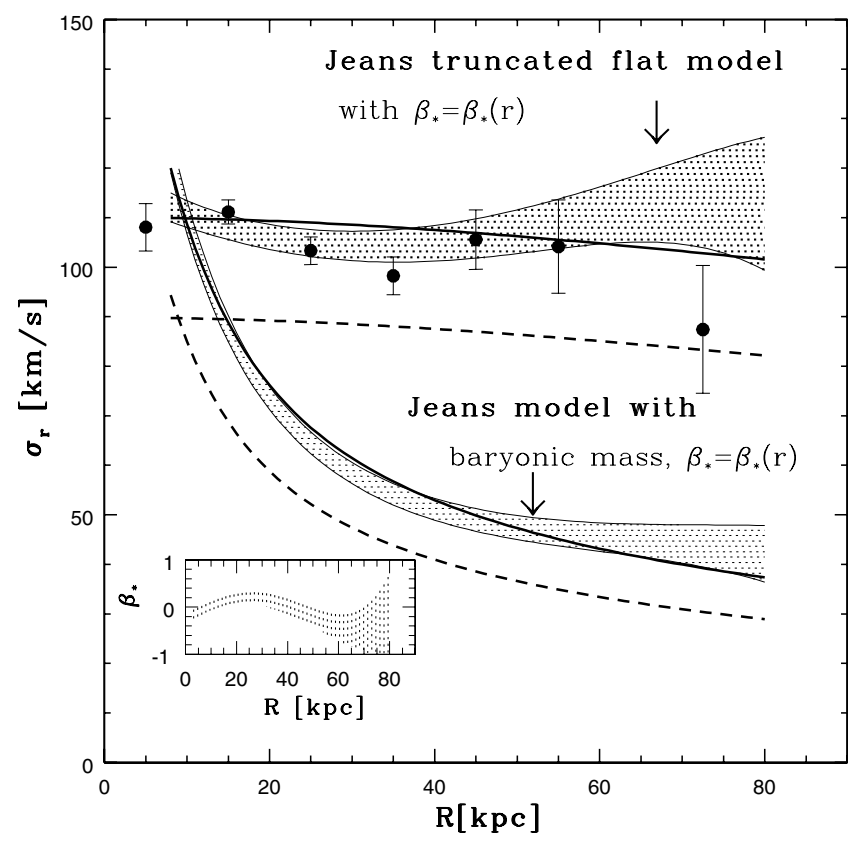

Fig. 3. Newtonian models based on the Jeans equation of the velocity dispersion of the BHB stars in the Milky Way. Lower hatched region is for the purely visible baryonic mass, and the upper hatched region is for the truncated flat model, which assumes the existence of dark matter. The widths of the regions are determined by uncertainties in establishing the anisotropies in the motion of BHB stars. The solid line is for the fit based on the isotropic case $\left(\beta_{*}=0\right)$, and the dashed line is for the purely radial case $\left(\beta_{*}=1\right)$ (in this and in all other Jeans models below). The inserted plot presents the $\beta_{*}$ parameter as a function of radius used in the Jeans models in this and all other figures related to the Jeans models below.

at large radii. In any case we also examine the limiting cases with $\beta_{*}=0$ and $\beta_{*}=1$, as typically done in the literature.

In both the Newtonian and the MOND approaches we use the following components of the mass:

- the bulge with total mass $M_{\text {bulge }}=1.5 \times 10^{10} M_{\odot}($ Xue08);

- the stellar disk with the total mass $M_{\text {stdisk }}=5 \times 10^{10} M_{\odot}$ (Xue08); and

- the gas disk with the total mass $M_{\text {gasdisk }}=1.18 \times 10^{10} M_{\odot}$ (McGaugh 2008).

The total disk mass is then $M_{\text {disk }}=M_{\text {stdisk }}+M_{\text {gasdisk }}=6.18 \times$ $10^{10} M_{\odot}$. In the Newtonian model we add a dark halo (see Sect. 3.1), and in the MOND model no additional matter is added in an attempt to fit the velocity dispersion.

\subsection{Newtonian models}

In the Newtonian models without dark matter, we adopted the form of the total potential (see Xue08),

$\Phi_{\text {tot }}(r)=\Phi_{\text {disk }}(r)+\Phi_{\text {bulge }}(r)$,

where the spherically symmetric potential for the bulge takes the form

$\Phi_{\text {bulge }}(r)=-\frac{G M_{\text {bulge }}}{r+c_{0}}$, 
and the spherically symmetric potential for the disk takes the form

$\Phi_{\mathrm{disk}}(r)=-\frac{G M_{\mathrm{disk}}\left(1-\mathrm{e}^{-\frac{r}{b}}\right)}{r}$,

where we took $c_{0}=0.6 \mathrm{kpc}$ and $b=4 \mathrm{kpc}$ (Xue08; see also Smith et al. 2007). In the analysis of the presumed dark matter contribution, we use the truncated flat model based on the extension of the Jaffe (1983) model:

$\Phi_{\mathrm{DM}}(r)=\frac{G M}{a} \log \left(\frac{\sqrt{r^{2}+a^{2}}+a}{r}\right)$

where $M$ is the total mass of the system and $a$ is the scalelength (Wilkinson \& Evans 1999). We chose this model for simplicity. The circular velocity in the model is given as

$V_{\mathrm{c}}^{2}(r)=\frac{V_{0}^{2} a}{\left(r^{2}+a^{2}\right)^{1 / 2}}$

where we adopted the value of $V_{0}=220 \mathrm{~km} \mathrm{~s}^{-1}$ in our modeling and for the scalelength, $a=105 \mathrm{kpc}$ as found in Battaglia et al. (2005).

The results of solving Eq. (4) using the aforementioned assumptions are given in Fig. 3. The shaded regions in Fig. 3 and in all figures related to the Jeans modeling are determined by the measured orbital anisotropies as given in Fig. 1 (lowest panel) and Table 1: the upper limit of each region is determined by the lowest value of the anisotropy and vice versa, the lower limit of each region is determined by the highest value of the anisotropy. In Fig. 3 the lower region only includes visible baryonic mass and the upper one is for the truncated flat model, which assumes the existence of dark matter. One can see the expected result in the lower shaded zone: visible baryonic mass alone cannot explain the kinematics of the external regions of the Milky Way. Using the measured anisotropies, one can see that a significant amount of additional (dark) matter is needed to obtain a fit to the observed velocity dispersion. Additionally, as mentioned earlier, we also tested two particular cases for all the models: isotropic case $\left(\beta_{*}=0\right)$ and purely radial case $\left(\beta_{*}=1\right)$. To fit the outermost point using the truncated flat model, one needs radial anisotropies. Including only anisotropies (without the existence of dark matter) in the Jeans equation cannot provide a successful fit of the velocity dispersion in the case of the Milky Way. Such a case, however, can be encountered when one models external regions of early-type galaxies (for example, the case of tangential anisotropies in the galaxy NGC 4649 for which dark matter is unnecessary even beyond $\sim 3$ effective radii, see Samurović \& Ćirković 2008).

\subsection{MOND models}

The MOND theory (Milgrom 1983) is widely used when one models rotation curves of the Milky Way (e.g. McGaugh 2008) and other spiral galaxies (e.g. Sanders 1996; Sanders \& McGaugh 2002; Gentile et al. 2011). In this section we solve the nonrotating Jeans equation, again in the spherical approximation, noting that the MOND theory is not insensitive to the massorbital anisotropy degeneracy, thus taking the measured orbital anisotropies into account. The Newtonian acceleration is given as $a_{\mathrm{N}}=a \mu\left(a / a_{0}\right)$, where $a$ is the MOND acceleration; here, $\mu(x)$ is the MOND interpolating function, with $x \equiv a / a_{0}$, and a universal constant $a_{0}=1.35_{-0.42}^{+0.28} \times 10^{-8} \mathrm{~cm} \mathrm{~s}^{-2}$ (Famaey et al. 2007). Thus, for $a \gg a_{0}$ the interpolation function $\mu\left(a / a_{0}\right) \approx 1$ leads to the Newtonian expression for the acceleration and for $a \ll a_{0}$ one has $\mu=a / a_{0}$. We applied several different MOND approaches for which we present here the pairs of equations (expressions for $\mu(x)$ and for the MOND circular velocity $V_{\mathrm{c}, \mathrm{M}}$ where $V_{\mathrm{c}, \mathrm{N}}$ is the Newtonian circular velocity in all the expressions below):

1. the "simple" MOND formula from Famaey \& Binney (2005):

$\mu(x)=\frac{x}{1+x}$

produces the following expression for $V_{\mathrm{c}}$ (e.g. Richtler et al. 2008):

$V_{\mathrm{c}, \mathrm{M}}^{2}=\frac{V_{\mathrm{c}, \mathrm{N}}^{2}}{2}+\sqrt{\frac{V_{\mathrm{c}, \mathrm{N}}^{4}}{4}+V_{\mathrm{c}, \mathrm{N}}^{2} \times a_{0} \times r} ;$

2. for the "standard" formula (Sanders \& McGaugh 2002),

$\mu(x)=\frac{x}{\sqrt{1+x^{2}}}$,

the circular velocity becomes (Samurović \& Ćirković 2008):

$V_{\mathrm{c}, \mathrm{M}}^{4}=\frac{V_{\mathrm{c}, \mathrm{N}}^{4}}{2}+\sqrt{\frac{V_{\mathrm{c}, \mathrm{N}}^{8}}{4}+V_{\mathrm{c}, \mathrm{N}}^{4} \times a_{0}^{2} \times r^{2}} ;$

3. for the Bekenstein's “toy” model (Bekenstein 2004),

$\mu(x)=\frac{-1+\sqrt{1+4 x}}{1+\sqrt{1+4 x}}$,

the circular velocity is (Samurović \& Ćirković 2008):

$V_{\mathrm{c}, \mathrm{M}}^{2}=V_{\mathrm{c}, \mathrm{N}}^{2}+\sqrt{a_{0} \times r} V_{\mathrm{c}, \mathrm{N}}$

4. we also tested the MOND model conceived by Zhao (2007), which was meant to explain the exact amount of dark energy. It belongs to the same family of MOND models as the "simple" model, namely

$\mu(x)=1-\left(1+\frac{x}{n}\right)^{-n}$.

The "simple" model is obtained for $n=1$, and the Zhao (2007) model is obtained for $n=3$. Calculation of the circular velocity because of the value of $n=3$ becomes much more complex so we solved it numerically. It is shown (with other circular velocity curves) in Fig. 8.

We solved the Jeans equation for all the MOND models assuming only visible baryonic mass and orbital anisotropies mentioned earlier, and the results are given below. We stress that there are no free parameters in the models that we present below. We take into account only the visible, baryonic mass found in the bulge and in the stellar and gas disks. The widths of the hatched regions in all the cases are again exclusively due to uncertainties of the anisotropies. We perform our modeling beyond $\sim 8 \mathrm{kpc}$ since the data in the innermost region are scarce. In a radius $\sim 5 \mathrm{kpc}$ we only have two BHB stars.

The "simple" MOND approach provides a very good fit to the observed velocity dispersion beyond $\sim 10 \mathrm{kpc}$ (Fig. 4). In the inner regions (inside $\sim 10 \mathrm{kpc}$ ), the predicted velocity dispersion 


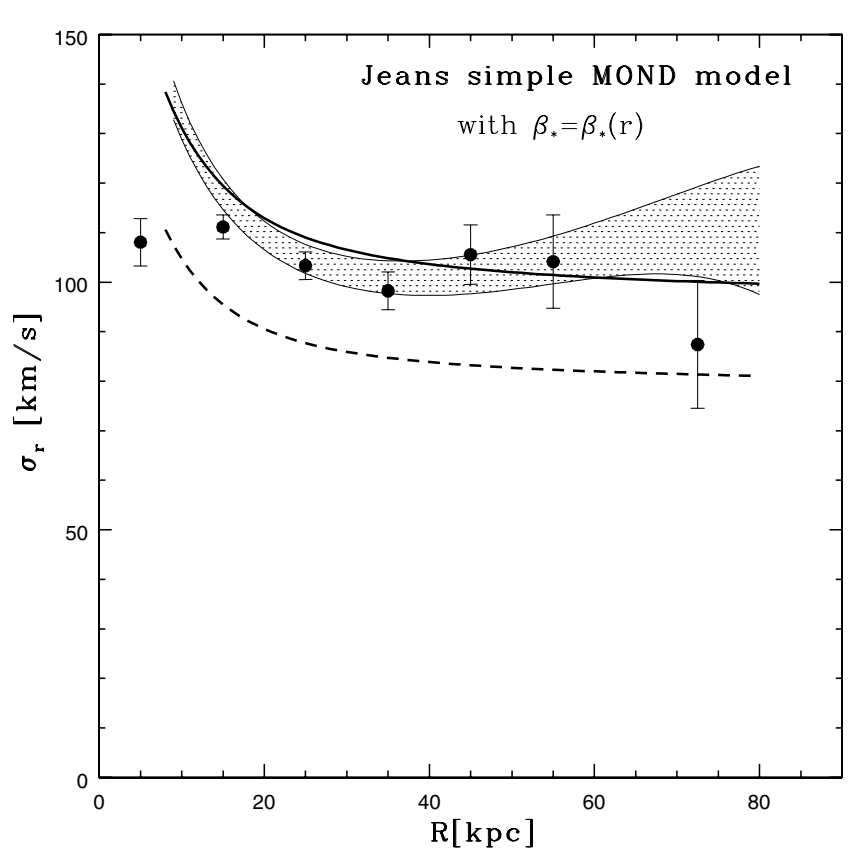

Fig. 4. Jeans modeling of the Milky Way using the "simple" MOND approach. The meaning of the symbols and curves is the same as in Fig. 3.

is too high (as in the case of the Newtonian model with the baryonic mass). The fit of the outermost point at $73 \mathrm{kpc}$ appears to be marginally too high, but given the small number of BHB stars and large uncertainty of the value of the velocity dispersion in the last bin, we do not consider this a serious problem. Overall, the "simple" MOND approach provides a satisfactory fit to the observed radial velocity dispersion beyond $\sim 20 \mathrm{kpc}$.

The "standard" MOND model, presented in Fig. 5, also provides a very good fit to the observed velocity dispersion beyond $\sim 10 \mathrm{kpc}$ and can even marginally fit the outermost velocity dispersion point. Note, however, the small discrepancy with the fit of the value at $25 \mathrm{kpc}$. Again, one can see that the "standard" MOND approach also provides a satisfactory fit to the observed radial velocity dispersion.

We tested the "toy" MOND model. In Fig. 6 one can see that in this MOND approach the fit of the observed velocity dispersion (apart from the region between 40 and $60 \mathrm{kpc}$ ) is not good (assuming the anisotropies shown in Fig. 3) and predicts too high a mass. The fits tend to improve if one assumes radial anisotropies $\left(\beta_{*}>0\right)$.

Finally, we tested the Zhao (2007) model and show the results in Fig. 7. This approach predicts a good fit of the observed velocity dispersion beyond $\sim 8 \mathrm{kpc}$. It provides an even moderately better fit than the "simple" MOND model, which belongs to the same family of models (see the innermost and the outermost regions). As in the case of the "simple" model it appears that radial anisotropies are needed in the outermost part (beyond $\sim 60 \mathrm{kpc}$ ) of the Milky Way.

It is interesting to compare our results with those from the paper by Angus et al. (2008) who analyzed the velocity distribution of Sloan Digital Sky Survey (SDSS) satellites in MOND. They used the "simple" MOND model and solved the Jeans equation in MOND to find that the two line-of-sight velocity dispersion profiles can be fitted with a radially varying anisotropy.

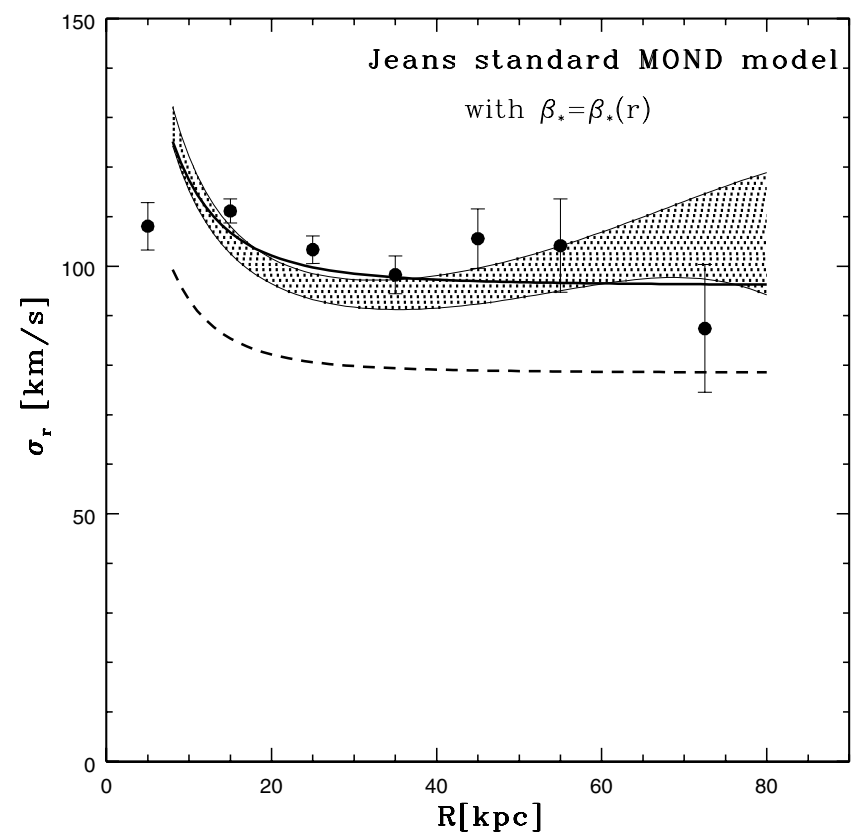

Fig. 5. Jeans modeling of the Milky Way using the "standard" MOND approach. The meaning of the symbols and curves is the same as in Fig. 3.

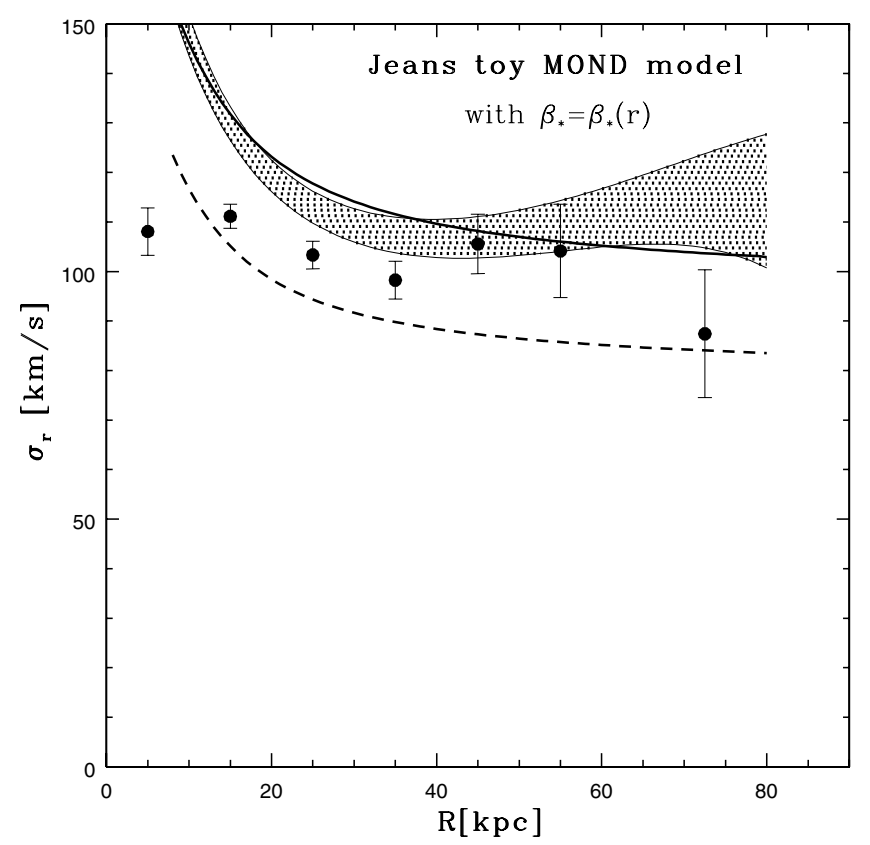

Fig. 6. Jeans modeling of the Milky Way using the "toy" MOND approach. The meaning of the symbols and curves is the same as in Fig. 3.

They find that the transition from more tangentially to more radially based orbits occurs at $\sim 50 \mathrm{kpc}$. From our Jeans model based on all tested MOND approaches (see Figs. 4-7), we see that in order to fit the outermost point at $\sim 85 \mathrm{kpc}$ one needs to introduce the radial anisotropies which agrees with the results of Angus et al. (2008). New observations of the tracers in 


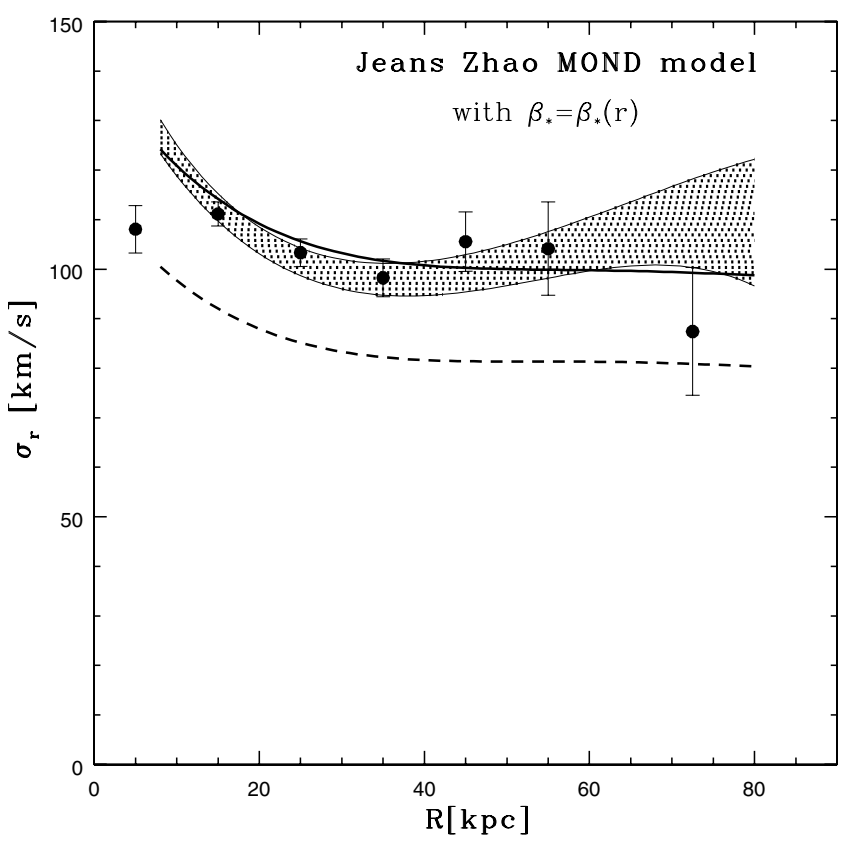

Fig. 7. Jeans modeling of the Milky Way using the Zhao (2007) MOND approach. The meaning of the symbols and curves is the same as in Fig. 3.

the Milky Way will provide better statistics and thus will impose more stringent constraints on the anisotropies in the outer regions of the Galaxy. It is worth repeating (see Sect. 3.1) that there are no anisotropies that would fit the observed velocity dispersions for the purely baryonic matter Newtonian model in the case of the Milky Way.

Overall, in this section it is shown on a well-studied example of our Galaxy that the Jeans modeling of galactic velocity dispersions is indeed a useful diagnostic tool for establishing the presence of dark matter (or the need for an alternative theory of gravity such as MOND).

\subsection{Circular velocity curves for the Newtonian and MOND models}

In this section we present the circular velocity curves $\left(v_{\mathrm{c}}=v_{\mathrm{c}}(r)\right)$ for all the models for which we perfomed the Jeans modeling above. This way of testing the theoretical models was used in $\mathrm{Wu}$ et al. (2008), who analyzed Milky Way potentials in cold dark matter and MOND approaches. Our aim now is to test all the models (both Newtonian and MOND) using a different methodology. Our estimate of the mass at a given radius is based on the tracer mass estimator (TME) formula from Watkins et al. (2010), which uses radial velocities $\left(v_{r}\right)$ of tracers at a given galactocentric distance $(r)$ :

$M(r)=\frac{C}{G}\left\langle v_{r}^{2} r^{\alpha^{\prime}}\right\rangle, \quad C=\left(\alpha^{\prime}+\gamma-2 \beta_{*}\right) r_{\mathrm{out}}^{\left(1-\alpha^{\prime}\right)}$.

In our estimates we assumed the isothermal case $\left(\alpha^{\prime}=0\right)$ with $\gamma=4$, where $\gamma$ is the slope of tracer density, and for simplicity and to make comparison with the models easier we only used the isotropic case $\left(\beta_{*}=0\right)$. In the estimates of error bars, only uncertainties in the distances are taken into account. Using the outermost point $\left(r_{\text {out }} \sim 85 \mathrm{kpc}\right.$ ) we found that the cumulative mass of the Milky Way inside that point is equal to

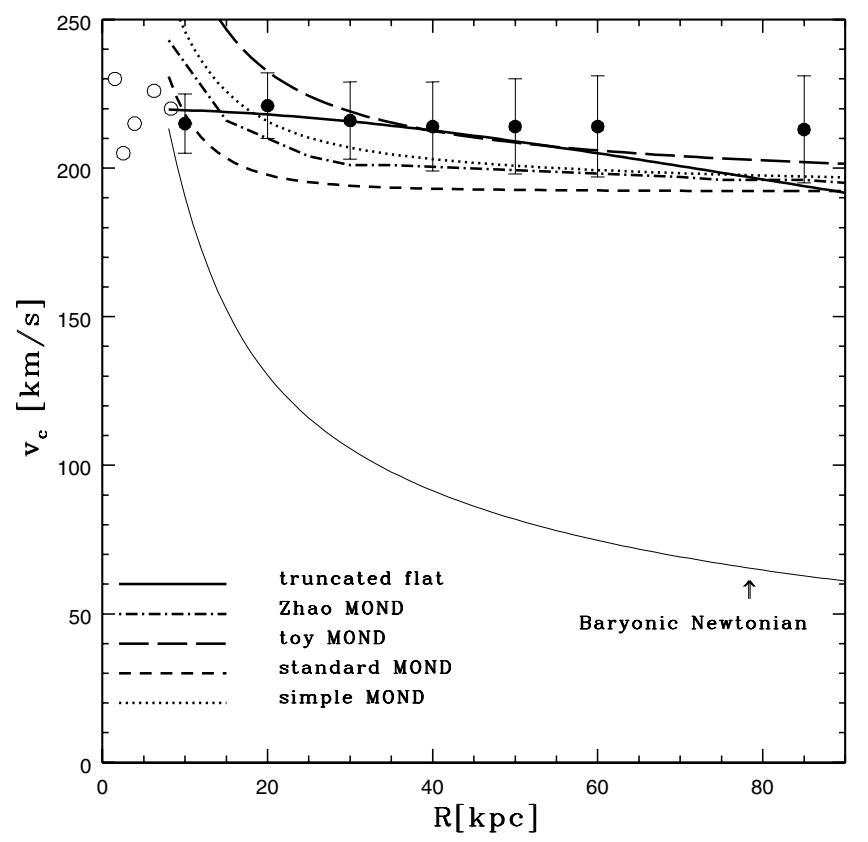

Fig. 8. Circular velocity curves of all the models tested in Sect. 3.2. The observed points are obtained using TME (see text for details). All the models and the observed points assume isotropy of the orbits. The data from the inner part of the Galaxy plotted using open circles are taken from Caldwell \& Ostriker (1981).

$(8.83 \pm 0.73) \times 10^{11} M_{\odot}($ in agreement with Gnedin et al. 2010), which corresponds to $v_{\mathrm{c}} \approx 213 \pm 18 \mathrm{~km} \mathrm{~s}^{-1}$. We note that the Milky Way rotation curve data do not extend beyond $\sim 12 \mathrm{kpc}$, and since we are interested in constraining our models at large radii, we do not require our models to fit the inner galaxy data. However, as a useful check of our adopted parameters, we plotted in Fig. 8 the rotation curve data (open circles) as presented in Wu et al. (2007, their Fig. 1).

From Fig. 8 one can see that the Newtonian purely baryonic model (as expected) cannot fit the data (except for the innermost point), whereas the truncated flat model that assumes a significant amount of dark matter provides a good fit to the circular velocity curve. The MOND models, "simple", "toy", and Zhao models provide a satisfactory fit beyond $\sim 20 \mathrm{kpc}$, whereas the "standard" MOND model provides a fit in the inner region, and beyond it shows departures from the observed points albeit not very strong ones. It is worth noting that the "simple" and "toy" MOND models (and to a lesser extent, also the Zhao model) along with simplified model of the total mass of the Milky Way predict too high a mass interior to $\sim 20 \mathrm{kpc}$ (or radial anisotropies there), and that beyond that point they fit the circular velocities. Taking both the Jeans modeling and the methodology of the circular velocity curves into account, one can see that the truncated flat model (with dark matter) and the MOND "simple" and Zhao (2007) models (without dark matter) provide good fits to the observed data. At this stage, the other two MOND models can provide a fit by assuming various anisotropies; therefore, only when the anisotropies are more firmly established can one begin the process of distinguishing between the models. The data and the models clearly exclude one model: the purely baryonic Newtonian model. 


\section{Conclusions}

We used a sample of 2557 BHB stars that extend out to $85 \mathrm{kpc}$ to investigate the kinematics of the Milky Way halo. We determined radial velocity dispersions, skewness, and kurtosis parameters and solved the Jeans equation taking orbital anisotropies into account.

Our conclusions are as follows.

1. The profile of the radial velocity dispersion is approximately constant throughout the whole Galaxy with $\sigma_{r} \approx$ $100 \mathrm{~km} \mathrm{~s}^{-1}$. The skewness parameter $s_{3}$ is either consistent with zero or is moderately positive. On the other hand, the kurtotic parameter $s_{4}$, apart from one point at $25 \mathrm{kpc}$, is consistent with zero and moderately negative. Since the number of stars per bin decreases the error bars inevitably increase in the outer bins. The future surveys will narrow them down thus imposing stronger constraints on the kinematics of the Milky Way galaxy in its outer regions.

2. We investigated the rotation of BHB halo stars and found that the rotation is negligible within a radius $\sim 30 \mathrm{kpc}$ and that beyond $\sim 30 \mathrm{kpc}$ the rotation amplitude changes from zero, although it remains very low $\left(-8.39 \pm 4.88 \mathrm{~km} \mathrm{~s}^{-1}\right)$. In our Jeans models, we therefore neglected the rotation.

3 . We solved the nonrotating Jeans equation in the spherical approximation in both the Newtonian and the MOND approaches. We recovered the well-known result that the kinematics of the Milky Way cannot be fitted without the additional (dark) matter. We tested the truncated flat model and found that it provides a good fit of the observed velocity dispersion, which implies a significant amount of dark matter in the Newtonian approach. We tested four MOND models: "simple", "standard", and Zhao (2007) models provide satisfactory fits of the observed velocity dispersion assuming isotropic orbits and the existence of visible baryonic matter alone, whereas Bekenstein's "toy" would need radial anisotropies for a satisfactory fit. We also tested the circular velocity curves for all the models: the "simple" and Zhao MOND models (which belong to the same family of models) provide the best fit without introducing anisotropies (although some radial anisotropies appear to be necessary in the innermost region), whereas other MOND models would need radial (the "toy" model) and tangential (the "standard" model) anisotropies in their outer parts. It is therefore worth noting that all four MOND models should be taken into account in the studies of galactic dynamics. We still need to analyze the difference between the MOND models of earlyand late-type galaxies in more detail in the future and the property of MOND to successfully fit spirals while failing to do so in the case of ellipticals (at least in some cases. For example, Tiret et al. 2007, find that a MOND model for the dynamics of NGC 3379 reproduces the observations on all scales, and on the other hand, Richtler et al. 2008, who investigated NGC 1399, claim that additional dark matter is needed for this galaxy).
Acknowledgements. The authors thank Slobodan Ninković for numerous interesting discussions. The authors acknowledge Warren Brown and Alis Deason for the clarifications of the work they did with their collaborators. This work was supported by the Ministry of Education and Science of the Republic of Serbia through project No. 176021, "Visible and Invisible Matter in Nearby Galaxies: Theory and Observations". The authors gratefully acknowledge the valuable comments of the anonymous referee, who helped to improve the quality of the manuscript and to correct the error present in its initial version.

\section{References}

Angus, G. W., Famaey, B., Tiret, O., Combes, F., \& Zhao, H. S. 2008, MNRAS, 383, L1

Battaglia, G., Helmi, A., Morrison, H., et al. 2005, MNRAS, 364, 433

Bekenstein, J. 2004, Phys. Rev. D, 70, 083509

Binney, J. J., \& Tremaine, S. 2008, Galactic Dynamics, second edition (Princeton: Princeton Univ. Press)

Brown, W. R., Geller, M. J., Kenyon, S. J., \& Diaferio, A. 2010, AJ, 139, 59 (Bro10)

Caldwell, J. A. R., \& Ostriker, J. P. 1981, ApJ, 251, 61

Deason, A. J., Belokurov, V., \& Evans, N. W. 2011, MNRAS, 411, 1480

Famaey, B., \& Binney, J. 2005, MNRAS, 363, 603

Famaey, B., Gentile, G., Bruneton, J.-P., \& Zhao, H. S. 2007, Phys. Rev. D, 75, 063002

Gentile, G., Famaey, B., \& de Blok, W. J. G. 2011, A\&A, 527, A76

Gerhard, O. 1993, MNRAS, 265, 213

Gnedin, O. Y., Brown, W. R., Geller, M. J., \& Kenyon, S. J. 2010, ApJ, 720, L108

Jaffe, W. 1983, MNRAS, 202, 995

Kendall, M., \& Stuart, A. 1977, The Advanced Theory of Statistics, Distribution Theory, fourth edition (London and High Wycombe: Charles Griffin \& Co. Ltd.), 1

McGaugh, S. M. 2008, ApJ, 683, 137

Milgrom, M. 1983, ApJ, 270, 365

Napolitano, N. R., Romanowsky, A. J., Capaccioli, M., et al. 2011, MNRAS, 411, 2035

Peng, E. W., Ford, H. C., \& Freeman, K. C. 2004, ApJ, 602, 685

Richtler, T., Dirsch, B., Gebhardt, K., et al. 2004, AJ, 127, 2094

Richtler, T., Schuberth, Y., Hilker, M., et al. 2008, A\&A, 478, L23

Romanowsky, A. J., Douglas, N. G., Arnaboldi, M., et al. 2003, Science, 5640, 1696

Sakamoto, T., Chiba, M., \& Beers, T. C. 2003, A\&A, 397, 899

Salucci, P., Martins, C. F., \& Lapi, A. 2011 [arXiv: 1102 .1184]

Samurović, S. 2010, A\&A, 514, A95

Samurović, S., \& Ćirković M. M. 2008, A\&A, 488, 873

Samurović, S., \& Danziger, I. J. 2005, MNRAS, 363, 769

Samurović, S., \& Danziger, I. J. 2006, A\&A, 458, 79

Sanders, R. H. 1996, 473, 117

Sanders, R. H., \& McGaugh, S. 2002, ARA\&A, 40, 263

Schuberth, Y., Richtler, T., Hilker, M., et al. 2010, A\&A, 513, A52

Smith, M. C., Ruchti, G. R., Helmi, A., et al. 2007, MNRAS, 379, 755

Sofue, Y., Honma, M., \& Omodaka, T. 2009, PASJ, 61, 227

Tiret, O., Combes, F., Angus, G. W., Famaey, B., \& Zhao, H. S. 2007, A\&A, 476, L1

Tonry, J. L. 1983, ApJ, 266, 58

Watkins, L. L., Evans, N. W., \& An, J. H. 2010, MNRAS, 406, 264

Wilkinson, M., \& Evans, N. W. 1999, MNRAS, 310, 645

Wu, X., Zhao, H. S., Famaey, B., et al. 2007, ApJ, 665, L101

Wu, X., Famaey, B., Gentile, G., Perets, H., \& Zhao, H. S. 2008, MNRAS, 386, 2199

Xue, X. X., Rix, H. W., Zhao, G., et al. 2008, ApJ, 684, 1143 (Xue08)

Zhao, H. S. 2007, ApJ, 671, L1 